\title{
Daniel Hildebrand Waldschlößchen ohne Brücke: über Aussetzung und Selbstausschaltung von Demokratie ${ }^{1}$
}

Ein Streit, der in Dresden seit längerem um den Bau einer Straßenbrücke geführt wird, hat im vergangenen Jahr mehrere Wochen lang überregionales Aufsehen erregt und wird möglicherweise noch ein verfassungsrechtliches Nachspiel haben. Er ist für die Krise nicht nur kommunaler Demokratie in Deutschland symptomatisch.

\section{Sachverhalt}

Nachdem bereits in den I990er Jahren Pläne für eine zusätzliche Elbquerung öffentlich erörtert und ein Planfeststellungsverfahren auf den Weg gebracht worden waren, entschieden sich in einem Volksentscheid, wie ihn $\ 24$ der Sächsischen Gemeindeordnung damals geltender Fassung vorsah, ${ }^{2}$ die Bürger am 27. Februar 2005 für den Bau eines »Verkehrszuges Waldschlößchenbrücke«, der eine Elbquerung in Gestalt einer Straßenbrücke vorsah.

Die UNESCO setzte am I I. Juli 2006 das Elbtal auf die sogenannte »rote Liste« des Weltkulturerbes und drohte zugleich an, diesen Status auf der nächsten Tagung im Jahre 2007 wieder zu entziehen, falls ein Brückenbauwerk wie das von den Bürgern gewünschte errichtet werde. 3 Daraufhin beschloss der Stadtrat am 20. Juli 2006, einem Antrag der Fraktion Bündnis 90/Die Grünen folgend, die unmittelbar bevorstehenden Bauarbeiten auszusetzen. Zugleich solle ein neuer Bürgerentscheid vorbereitet werden. ${ }^{4}$ Am 26. Juli legte der stellvertretende Bürgermeister dagegen Widerspruch beim Rat ein,'s der am Io. August seinen Beschluss vom 20. Juli bestätigte, ${ }^{6}$ woraufhin der Oberbürgermeister am I I. August 2006 erneut Widerspruch beim Stadtrat einlegte. Mit selbem Schreiben erklärte er jedoch zudem, er »werde unverzüglich die Entscheidung des Regierungspräsidiums Dresden über die« seiner Meinung nach fehlende »Rechtmäßigkeit der Beschlüsse herbeiführen «.7 Das Regierungspräsidium stellte am I4. August die Rechtswidrigkeit des Ratsbeschlusses vom io. August fest. Der Beschluss hindere den Oberbürgermeister, seiner Verpflichtung nachzukommen, dem Bürgerentscheid vom 27. Februar 2005 »Geltung zu verschaffen « 8 Diese Erkenntnis zeitigte aus Sicht des Regierungspräsidiums zwei Rechtsfolgen: Zum einen müsse der Stadtrat seinen Beschluss aufheben und zum anderen eine Vergabeentscheidung treffen. Dafür setzte das Regierungspräsidium dem Rat eine Frist bis zum 24. August und drohte bei Nichterfüllung Ersatzvornahme an. ${ }^{9}$ Zum letztmöglichen Termin, nämlich am 24. August, versuchte der

I Der Autor dankt Frau Dr. iur. Sonja Mühlenbruch, Universität Bremen, für ihre Beratung.

$2 \int 23$ Sächsische Gemeindeordnung: »(I) In Gemeindeangelegenheiten können die Bürger über eine zur Abstimmung gestellte Frage entscheiden (Bürgerentscheid), wenn ein Bürgerbegehren Erfolg hat. [...] (2) Der Bürgerentscheid kann über alle Fragen durchgeführt werden, für die der Gemeinderat zuständig ist. «

3 Eine solche Konfliktlage war bereits, bevor der Volksentscheid im Jahre 2005 stattfand, in der öffentlichen Diskussion um die Bewerbung des Elbtals als Weltkulturerbe erörtert worden.

4 Beschluss A 0308-SR 35-06 des Rates der Landeshauptstadt Dresden vom 20. Juli 2006.

5 Schreiben des Oberbürgermeisters an die Mitglieder des Rates vom 26. Juli 2006.

6 Beschluss V I 368-SR 36-06 des Rates der Landeshauptstadt Dresden vom Io. August 2006.

7 Schreiben des Oberbürgermeisters an die Mitglieder des Rates vom I I. August 2006, kein Aktenzeichen.

8 Zit. nach Regierungspräsidium Dresden Pressemitteilung 40/2006 vom I 4. August 2006.

9 Ebd. 

Oberbürgermeister beauftragte, den Planfeststellungsantrag, den »Verkehrszug Waldschlößchenbrücke« zu errichten, zurückzuziehen. Darüber hinaus beauftragte er den Oberbürgermeister, der ja erst durch seinen Widerspruch gegen den Beschluss vom Io. August den erneuten Beschluss vom 24. August herbeigeführt hatte, gegen die für diesen Fall angedrohte Ersatzvornahme durch das Regierungspräsidium oder durch einen Dritten Widerspruch beim Regierungspräsidium einzulegen. ${ }^{\circ}{ }^{\circ}$ Am 25 . August ordnete daraufhin das Regierungspräsidium die

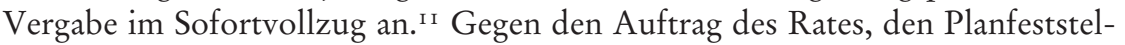
lungsantrag zurückzuziehen, legte der Oberbürgermeister am selben Tag, aber offensichtlich gleichzeitig oder unmittelbar nach der Anordnung des Regierungspräsidiums Widerspruch beim Stadtrat ein. Gegen den Auftrag des Rates, Widerspruch beim Regierungspräsidium gegen die drohende und inzwischen auch eingetretene Ersatzvornahme des Regierungspräsidiums Widerspruch einzulegen, konnte der Oberbürgermeister keinen Widerspruch einlegen. ${ }^{\mathrm{I} 2}$ Daher legte er fristgerecht gegen die Ersatzvornahme des Regierungspräsidiums Widerspruch ein, über den das Verwaltungsgericht Dresden zu entscheiden hatte. Dieses setzte die Ersatzvornahme am 28. August 2006 durch Zwischenentscheid und am 30. August 2006 durch Beschluss aus. ${ }^{13}$ Dem Antrag der Vertreter des Bürgerbegehrens, beigeladen zu werden, gab das Verwaltungsgericht nicht statt. ${ }^{\mathrm{I}}{ }^{4}$ Gegen den Beschluss des Verwaltungsgerichts, die Ersatzvornahme auszusetzen, legte das Regierungspräsidium am I. September 2006 beim sächsischen Oberverwaltungsgericht in Bautzen Beschwerde ein. ${ }^{\text {Is }}$

Am 8. November entschied der zuständige 4. Senat des Oberverwaltungsgerichtes im Rahmen eines nichtöffentlichen Erörterungstermins, ein Mediationsverfahren durchzuführen. ${ }^{16}$ Als Begründung wurde nun erstmals in

Io Beschluss V I378-SR 37-06 des Rates der Landeshauptstadt Dresden vom 24. August 2006.

I I Zit. nach RP Dresden Pressemitteilung 42/2006 vom 25. August 2006.

I 2 Zit. nach Schreiben des Oberbürgermeisters der Landeshauptstadt Dresden an den Rat vom 25. August 2005. Der Oberbürgermeister gerät in eine Lage, die für den einzelnen Bürger schon früh beschrieben worden ist: Die Institutionen Bürgerentscheid und Stadtrat drängen nämlich »auf miteinander unvereinbare Verhaltensschablonen«; Benjamin Akzin, Die Struktur von Staat und Recht. Eine Analyse, in: Der Staat 3 (1964), S. 26I (265).

I 3 Verwaltungsgericht Dresden AZ i 2 K i768/06.

I4 Ebd., Begründung auf S. 8 ff. Dies ist insofern erheblich, als ein einzelner Bürger, wenn er sich »in seinen Rechten aus $\ 24$ verletzt« sieht, gegen eine »Beanstandung des Bürgerentscheids durch die Rechtsaufsichtsbehörde wegen Rechtswidrigkeit oder Mißachtung der Sperrwirkung durch die Gemeindeorgane« verwaltungsgerichtliche Klage erheben kann, S. I 3 f. Einen potentiellen Kläger noch nicht einmal beizuladen, spricht weder für Konzilianz noch für weitsichtige Prozessökonomie.

Is Zit. nach RP Dresden Pressemitteilung 43/2006 vom I. September 2006.

I6 Die Krise kommunaler Demokratie wird nicht zuletzt darin symptomatisch, dass erst ein Gericht auftreten muss, um mit dem »Mediationsverfahren« ein originär demokratisch-partizipatorisches Verfahren anzuregen; Arno Waschkuhn, Demokratietheorien. Politiktheoretische und ideengeschichtliche Grundzüge, München I998, S. I33; diesen partizipatorischen Charakter der Mediation betont auch Susanne Nothhafft, Partizipation durch Mediation. Wege zur Konfliktlösung in der Zivilgesellschaft, München 2004, v. a. S. I3; zur exekutiven Funktion: Anna Geis, Regieren mit Mediation, Wiesbaden 2005; zum Verhältnis von Mediation und Recht: Stephan Breidenbach, Mediation. Struktur, Chancen und Risiken von Vermeidung im Konflikt, Köln I995, v.a. S. 77 ff. und ıо I ff.; Adriane Gullo, Mediation unter der Herrschaft des Rechts, Berlin 2006; Alexander Trunk, Schiedsgerichtsbarkeit und andere Formen alternativer Streitbeilegung, Berlin 2006; zur »bisher [...] nahezu unbekannten« Anwendung von Mediation speziell im Widerspruchsverfahren: Daniel Rapp, Mediation im Verwaltungsrecht - Möglichkeiten der Integration mediativer Elemente in das Widerspruchsverfahren, Tübingen 2004; Karsten Ortloff, Mediation im Verwaltungsprozess: Bericht aus der Praxis, Neue Zeitschrift für Verwaltungsrecht 2006, S. I48 ff. (I48); s. auch: Antje Kanngießer, Mediation zur Konfliktlösung bei Planfeststellungsverfahren, Hamburg 2004; Roland Fritz, Mediation statt Verwaltungsprozess?, München 2004; zur sozialen Praxis der Mediation allgemein: Elke Müller, Gerechtigkeitskonflikte in der Mediation, Hamburg 2004; Bernfried Kuthe, Verhandeln als innovativer Problemlösungsprozess, Aachen 2005; Esther Haas/Toni Wirz, Mediation, Zürich 2006. 
einer gerichtlichen Äußerung in dieser Angelegenheit eine Kollision kommunaler Demokratie mit völkerrechtlichen Verpflichtungen festgestellt. Neben den Verfahrensgegnern nahmen auch Vertreter der UNESCO an dem Termin teil. Vertreter des Bürgerbegehrens waren offensichtlich erneut nicht beteiligt. Die Prozessbeteiligten kamen daraufhin überein, das Gerichtsverfahren ruhen zu lassen. Das Mediationsverfahren wurde am 24. Januar 2007 für gescheitert erklärt. Das Regierungspräsidium beantragte am Tag darauf beim Oberverwaltungsgericht, das Verfahren wiederaufzunehmen. ${ }^{17}$ Das Oberverwaltungsgericht Bautzen hat am 9. März 2007 der Beschwerde des Regierungspräsidiums Dresden vom I. September stattgegeben, die vom Verwaltungsgericht angeordnete Aussetzung der Ersatzvornahme zu dispensieren..$^{18}$ Begründet wird dies mit dem überwiegenden öffentlichen Interesse, das im Vollzug des Bürgerentscheids liege, da der Ausgang der völkerrechtlichen Streitfragen nicht abzusehen sei. ${ }^{19}$ Die Mehrheit des Stadtrates will nun den verfassungsrechtlichen Weg beschreiten.

\section{Demokratietheoretische Analyse}

Bei der demokratietheoretischen Analyse der Affäre »Waldschlößchenbrücke« werden im Folgenden auch die völkerrechtlichen Implikationen insoweit behandelt, als sie für das Verständnis des innerstaatlichen Konfliktes erheblich sind. Im Oktober 2005, also nach dem Bürgerentscheid, wurde klar, dass die UNESCO ihre erste, bereits auf das Jahr 2004 datierende Aufnahme des Dresdner Elbtals auf Grundlage unzutreffender Vorstellungen darüber, wie eine Brükke die »Sichtbeziehungen « verändern oder einschränken würde, getroffen hatte. ${ }^{20}$ Bis heute umstritten ist, bei wem, Aufnahme beantragender Stadt Dresden oder UNESCO, die Ursache liegt. Der Stadtrat argumentiert, es bestehe eine völkerrechtliche Verpflichtung in Gestalt unmittelbar geltenden nationalen Rechts und stützt sich auf ein Gutachten des Dresdner Juristen Fastenrath. ${ }^{21}$ Die Brückenbefürworter sehen diese bindende Wirkung gerade nicht und berufen sich dabei auf ein Gutachten des Kölner Juristen Schöbener, demzufolge der Bund durch den Vollzug des Bürgerentscheids nicht vertragsbrüchig zu werden drohe. ${ }^{22} \mathrm{Zu}$ bemerken ist, dass die völkerrechtlich letztlich entscheidenden Institutionen, nämlich Bundesregierung, Bundestag und Bundesrat, sich erklärtermaßen aus dem Konflikt herausgehalten und sogar als Schlichter angeboten haben. ${ }^{23}$ Argumentiert wird also mit einer Bundestreue, auf der deren Empfänger anscheinend gar nicht besteht. Damit bleibt die Angelegenheit faktisch ein innerstaatlicher Konflikt, auch wenn er von Seiten des Stadtrates mit völkerrechtlichen Argumenten ausgetragen wird. Mehr noch: Sollte sich beim Stadtrat eine

I7 RP Dresden Pressemitteilung 08/2007 vom 25. Januar 2007.

I 8 Oberverwaltungsgericht Bautzen: Beschluss Az.: 4 BS 216/06, 9. März 2007.

I9 Oberverwaltungsgericht Bautzen: Beschluss Az.: 4 BS 2 16/06, 9. März 2007, S. I 3 f.

20 Deutsche UNESCO-Kommission e.V.: Das Elbtal in Dresden (www.unesco.de/320.html [Abruf 21. März 2007]).

2 I Kurzgutachtliche Stellungnahme zu Erwerb und Verlust des Welterbestatus (I I. Juli 2006) und Rechtsgutachtliche Stellungnahme zur Zulässigkeit des Baus der Waldschlösschenbrücke (9. August 2006) (=http://tu-dresden.de/die_tu_dresden/fakultaeten/juristische_fakultaet/jfoeffl $3 /$ news/welterbestatus.pdf [Abruf 2r. März 2007]).

22 Prof. Dr. Burkhard Schöbener: Gutachtliche Stellungnahme, 20. September 2006 (=www.pro-waldschloesschenbruecke.de/content/Rechtsgutachten_WSB_Prof_Schoebener.pdf [Abruf 2 I. März 2007]).

23 www.pro-waldschloesschenbruecke.de/content/29.09.2006\% 20Waldschloesschenbruecke-I.pdf (Abruf 2 I. März 2007); Bundestagsdebatte am 2. März 2007, BT-Drs. I6/44 I I. 
Argumentation einstellen, die die Haltung des Bundes als für die Geltung uner-

heblich ansieht, dann stellte sich der Dresdner Stadtrat letztlich sogar über den Bundestag. Das Gutachten von Fastenrath gibt dazu keine Auskunft, da es zu einem Zeitpunkt verfasst wurde, als der Bund seine schlichtende Position noch nicht erklärt hatte. Wohlweislich hat sich der Stadtrat, nachdem die Haltung des Bundes deutlich wurde, nicht dazu geäußert, ob das Recht auch gegen den Willen dessen, dessen Interessen gewahrt werden sollen, gilt. Auf der Seite der Brükkenbefürworter hingegen ging das Gutachten von Schöbener weiter, nämlich so weit, dem Bund tatsächlich das Recht abzusprechen, er könne allein durch Verträge kommunale Demokratie aussetzen. Hierzu hätte er vielmehr ein Gesetz erlassen müssen. Schließlich ist mittlerweile sogar der UNESCO selbst vorgeworfen worden, innerstaatliche Demokratie zu missachten, ${ }^{24}$ da sie ja bei ihrer Aufnahme des Elbtals in die »Rote Liste«, die im Sommer 2006 junktimiert mit der Aufgabe der Bauabsichten erfolgte, von dem entgegenstehenden Bürgerentscheid wusste: Das Argument des Stadtrates, der Inhalt des Bürgerentscheids sei aufgrund bindenden Völkerrechts rechtswidrig, wird also von der Gegenseite gespiegelt. Der Beschluss der UNESCO selbst sei rechtswidrig, weil er die Prinzipien innerstaatlicher Demokratie missachte. Von technischen Alternativüberlegungen wie einer Elbquerung mittels Tunnels soll an dieser Stelle abgesehen werden, obwohl diese als Optionen selbstverständlich weiter bestehen, aber nicht Teil des hier zu untersuchenden Problems sind.

Zunächst soll die faktische systembedingte Aufhebung von Demokratie betrachtet werden und im Folgenden e contrario gezeigt werden, dass Demokratie einen Absolutheitsanspruch in sich birgt, der auch nur durch einen institutionellen Absolutismus befriedigend und störungsfrei realisiert werden kann. Schließlich soll in einem weiteren Schritt die Funktion und Machtstellung der Judikative betrachtet werden. Dass sich diese Probleme verschränken und bedingen, die Teilung also rein arbeitsökonomischer Art ist, dürfte als evident zu erachten sein.

\section{Selbstausschaltung konkurrierender demokratischer Entscheidungsmodi}

Dass der Volkswille vom Rat, also dem kommunalen Parlament, missachtet wurde, erscheint zunächst eindeutig. Der mögliche Einwand, die Geschäftsgrundlage sei durch die UNESCO-Drohung entfallen, betrifft ein prinzipielles Dilemma von Volksentscheiden, aber letztlich jeder politischen, nämlich, gemäß Luhmann, kollektiv bindenden Entscheidung. ${ }^{25}$

Dass es der Bürgermeister, also ein Organ der Exekutive, und letztlich das Oberverwaltungsgericht, also ein Organ der Judikative, waren, die dem Volkswillen zur, wenn auch im Falle des OVG nur sehr eingeschränkten, Geltung verhalfen, heilt das demokratietheoretische Manko nicht. Zwar berief sich der (zweite) Bürgermeister in der Begründung seines Einspruchs ausdrücklich auf die Rechtswidrigkeit des Stadtratsbeschlusses, aber faktisch heilt dies nicht mehr den Autoritätsschaden des Souveräns. Dieser bleibt desavouiert, was vom Verwaltungsgericht in seinen späteren Urteilen noch faktisch sanktioniert wird. Durch den Beschluss des Stadtrates ist die unmittelbare Demokratie endgültig

24 So äußert sich namentlich der Bundestagsabgeordnete Jan Mücke in den Dresdner Neueste Nachrichten: »Waldschlößchenbrücke: Stadtrat stimmt gegen sofortigen Bau« vom 2 I. Juli 2006.

$25 \mathrm{Ob}$ die Bürger unter veränderter Informationslage überdies tatsächlich anders entschieden hätten, bleibe dahingestellt, repräsentative Umfragen lassen indes selbst daran zweifeln. 
ausgesetzt. Fortan sind die Entscheidungen obrigkeitlich und subordinierend, nicht kooperativ und koordinierend. ${ }^{26}$

Gegen diese Sicht der Dinge lässt sich nun die Einrede erheben, das Parlament sei schließlich gewählt und als solches unmittelbar demokratisch legitimiert. Unter den Bedingungen, wie sie die sächsische Kommunalverfassung normiert, lässt sich dagegen freilich die Gegeneinrede erheben, dass auch der Bürgermeister vom Volk direkt gewählt ist. All dies ändert jedoch nichts daran, dass der ausdrücklich erklärte Volkswille, der nun einmal Ergebnis eines Mehrheitsentscheides ist und eine Sperrwirkung von drei Jahren entfaltet, nicht unmittelbar wirkt. ${ }^{27}$ Als Mehrheitsprinzip praktizierte Demokratie kann keine derart konkurrierenden Institutionen vertragen. Ist eine von diesen, wie im vorliegenden Fall, zudem noch der Legislative, also der Volksvertretung zugehörig, kommt es gleichsam zu einer Selbstaufkündigung demokratischen Entscheidens. Da es jedoch nicht möglich ist, jede Sachentscheidung dem Volk vorzulegen, und solcherart demokratische Praxis in der Moderne auch nirgends anzutreffen ist, bedarf es immer repräsentativer, also parlamentarischer Elemente. ${ }^{28}$ Präsidiale hingegen, wie sie in der vorliegenden Konstellation der Bürgermeister darstellt, sind durchaus verzichtbar, ja sie tragen eher zu Friktionen bei. ${ }^{29}$ Der Fall der Dresdner Waldschlößchenbrücke zeigt, so paradox diese Aussage im konkreten Zusammenhang wirken mag, dass Demokratie störungsfrei nur parlamentarisch, also gemäß dem sogenannten »Westminstertypus «, auf keinen Fall aber durch verschiedene (potentiell) konkurrierende Institutionen und Funktionen bestehen kann: ${ }^{\circ}$ Denn diese geraten über kürzer oder länger notwendig mit einander in Konflikt, wie dies im

26 Zu dieser Begriffspolarität s. für viele Volker Neumann, Freiheitsgefährdung im kooperativen Sozialstaat. Rechtsgrundlagen und Rechtsformen der Finanzierung der freien Wohlfahrtspflege, Köln I992, S. 432, und die gesamte Festschrift von Berg et al. (Hrsg.), Regulierte Selbstregulierung als Steuerungskonzept des Gewährleistungsstaates. Ergebnisse des Symposions aus Anlass des 60. Geburtstages von Wolfgang Hoffmann-Riem, Berlin 200I. Mit Karl-Rudolf Korte/ Manuel Fröhlich, Politik und Regieren in Deutschland. Strukturen, Prozesse, Entscheidungen, Paderborn 2004, S. 245, ließe sich Koordination auch als Form »stillen Regierens « beschreiben: Wie auch immer Kortes Thesen zu bewerten sind, der vorliegende Untersuchungszusammenhang bietet ein Musterbeispiel für das, was »stilles Regieren« nicht ist.

$27 \ 24$ Abs. (4) SächsGO »Der Bürgerentscheid steht einem Entschluss des Gemeinderates gleich. Er kann innerhalb von drei Jahren nur durch einen neuen Bürgerentscheid abgeändert werden.« Dazu äußert der einschlägige Kommentar von Georg Brüggen/Ingrid Heggendorf, Sächsische Gemeindeordnung. Kommentar, Berlin I994: »Der Bürgerentscheid wirkt wie ein endgültiger Gemeinderatsbeschluss. Innerhalb von drei Jahren ist seine Abänderung nur durch einen neuen Bürgerentscheid möglich, so dass die einmal durch Bürgerentscheid getroffene Entscheidung selbst dann nicht geändert werden kann, wenn sich die rechtlichen oder tatsächlichen Verhältnisse geändert haben.« Der Stadtrat ist demnach also gänzlich außen vor.

28 Auch das am anderen Pol des Kontinuums demokratischer Systemalternativen stehende Modell der Konsensdemokratie, wie es die Schweiz repräsentiert, kommt nicht ohne repräsentative Institutionen aus, Arend Lijphart, Patterns of Democracy. Government Forms and Performance in Thirty-Six Countries, New Haven/London I999, S. 34 f. Vielmehr scheinen gerade Systeme, die auch plebiszitärer Demokratie vergleichsweise starkes Gewicht zubilligen, zugleich diejenigen zu sein, die auf die Repräsentativität von Demokratie stärkeren Wert legen. Zwar folgt die Dichotomie von »majoritarian democracy « und »consensus democracy « primär dem Kriterium der Mehrheitsdefinition und nicht demjenigen des Direktheitsgrades von Demokratie. Auffallend ist jedoch, dass je stärker ein System dem »majoritarianism « zuneigt, es direkter Demokratie desto weniger Bedeutung gibt.

29 Lijphart (Fn. 28), S. 9.

30 Wenn dieser Begriff hier verwandt wird, so zielt dies vornehmlich auf Parlamentsabsolutismus als leitendes Merkmal ab. Zwar ist das weitere Merkmal des »majoritarianism« nicht derart zwingend erforderlich, um Demokratie zu sichern. Aber es ist keinesfalls zufällig, sondern sachlich bedingt, dass nur ein System mit solchem Mehrheitsverständnis dem Gedanken der Mehrheitsherrschaft am reinsten entgegenkommen kann. Denn jedes andere Mehrheitsverständnis, also etwa dasjenige des Mehrheit absolut definierenden "pluralitarianism «, ist auf überproportional starke Beteiligung von Minderheiten angewiesen, Lijphart (Fn. 28), S. 9 f. »Absence of judicial review « stellt aber in jedem Falle ein konstitutives Merkmal dar, ebd., I9 f. Anzumerken ist, dass der Westminstertypus weltweit rückläufig ist. Dies führt freilich nicht überall zu einer Stärkung von Institutionen, die nicht vom Volk gewählt sind, wie vor allem das Beispiel der neuseeländischen Veränderungen zeigt, ebd., 2 I ff. Vielmehr bleibt gerade diesen beiden polaren Modellen gemeinsam, gegenüber judizieller Kontrolle weiterhin abgeneigt zu sein, s. Lijphart ebd., S. 4I. 

avouierung des Volkes, also des Souveräns, ist kein unglücklicher Zufall, sondern in der Eigenart der direkten Demokratie angelegt. Der schweizerische Archetypus direkter Demokratie, der oft als Beleg für die Praktizierbarkeit dieser Entscheidungsform angeführt wird, funktioniert vielmehr selbst nur höchst gestört. ${ }^{3}$ Demokratie neigt ihrer Anlage nach zu Monokratie.

\section{Störungen der Gewaltenteilung}

Neben diesem Konfliktdreieck, das sich noch zwischen Volk und unmittelbar von ihm legitimierten Institutionen vollzieht, besteht aber noch das in der öffentlichen Diskussion weithin beschwiegene Problem der dritten Gewalt. ${ }^{22}$

Erstinstanzlich hat diese vollkommen unverhohlen den Volkswillen außer Kraft gesetzt. Weder formell noch materiell ist dieser Beschluss gerechtfertigt. Selbst wenn die Judikative den Beschluss des Regierungspräsidiums hätte bestehen lassen und somit faktisch den Volksentscheid realisiert hätte, bleibt das Problem nicht nur rechtlich bestehen, sondern wird demokratietheoretisch noch brisanter: Denn der Oberbürgermeister hat der sächsischen Staatsregierung die Verantwortung übergeben, so dass nicht mehr die Umsetzung des Volksentscheides unmittelbarer Gegenstand ist. Die Initiatoren des Bürgerentscheids nicht zuzulassen, hat also eine gewisse Konsequenz - eine Konsequenz, die indessen verheerend für die Demokratie ist. Das Volk bleibt ein Volk von Regierungs- und Richters Gnaden. Wenn die vox populi die vox Dei wäre, gäbe es gar keine Kompetenzen mehr zu übergeben oder auch nur Entscheidungskompetenzen oder Entscheidungsinhalte gerichtlich zu prüfen. 33

Die Judikative ist nicht mehr eine rein gesetzesanwendende neutrale, sondern eine arbiträre übergeordnete Macht. Demokratie verträgt jedoch per definitionem nichts Übergeordnetes mehr: populus locutus causa finita. Kennzeichnend für den zeitgenössischen Demokratiediskurs ist indes, die Legitimität »unabhängiger« Institutionen darüber zu definieren, dass in bestimmten Politikfeldern demokratische Entscheidungsfindung nicht mehr ergebnisoffen sei, was freilich sachverständige Entscheidung gerade nicht sein darf, weil diese sich ja eben darüber legitimiert, eine vom Mehrheitsprinzip unabhängige »Wahrheit « zu vertreten. Dass demokratische Entscheidungsfindung vor allem im wirtschafts- und finanzpolitischen Feld nicht ergebnisoffen sein könne, ist dabei heute leitendes Paradigma.34 Darüber hin-

3I Im vorliegenden demokratietheoretischen Untersuchungszusammenhang ist dabei nicht in erster Linie die Ökonomie als vielmehr der Volkswille Maßstab: Dieser nehme jedoch durch die gesteigerte Macht von Interessengruppen an Kraft ab, wie etwa bei Henner Kleinewefers, Die direkten Volksrechte in der Schweiz aus ökonomischer Sicht, in: Silvio Borner/Hans Rentsch (Hrsg.), Wieviel Demokratie verträgt die Schweiz? Kritische Beiträge zur aktuellen Reformdebatte, Rüegger 1997, S. 6i (66) zu lesen ist; s. auch ders., ,Verwesentlichung < der Politik durch Einschränkung der direkten Volksrechte?, Freiburg (CH) I996, sowie Hans-Urs Wili, Jux Populi? Vox Dei? ıoo Jahre eidgenössische Volksinitiative auf Partialrevision der Bundesverfassung, in: Zeitschrift für schweizerisches Recht I991, S. 485 ff. Bei der bibliographischen Angabe »Jux« statt »vox« handelt es sich nicht um ein Versehen, was das Ausmaß an Erbitterung der Schweizerischen Debatte um direkte Demokratie exemplarisch veranschaulicht.

32 Zum Problem der Justiz für die Gewaltenteilung s. in der aktuellen Literatur z. B. Stefan Schieren, Gesetzgebung und Gerichtspraxis - ein Beitrag zur Gewaltenteilung, in: Politische Bildung 3 (2005), S. $40 \mathrm{ff}$.

33 Lijphard (Fn. 28), S. 19, 24 und 4 I.

34 V. a. James Buchanan, Die Grenzen der Freiheit, Tübingen 1984; zurückhaltender Giandomenico Majone, Independence vs. Accountability? Non-majoritarian institutions and democratic government in Europe, in: European University Institute working papers in political and social sciences, Florenz I994; ders., Nonmajoritarian institutions and the limits of democratic governance: A political transaction-cost approach, in: Journal of Institutional and Theoretical Economics $200 \mathrm{I}, \mathrm{S}$. 57 ff.; ders., Redistributive und sozialregulative Politik, in: Markus Jachtenfuchs/ Beate Kohler-Koch (Hrsg.), Europäische Integration, Opladen 32006, S. 225 ff.; Stephan Bredt, Die demokratische Legitimation unabhängiger Institutionen, Tübingen 2006, S. $280 \mathrm{ff}$. und passim. 
aus wird demokratisch verantwortlichen Entscheidungen eine Neigung zur Verschuldung unterstellt. Dass ausgerechnet Dresden eine der, zugegeben wenigen, Kommunen ist, die mittlerweile schuldenfrei ist, macht das vorliegende Beispiel nur zusätzlich pikant. Denn auch diese Legitimation allmählicher, aber zunehmend weniger diskreter Entdemokratisierung widerlegt das Beispiel Waldschlößchenbrücke. Wurde der Wahlkampf im Vorfeld des Bürgerentscheides hauptsächlich unter Abwägung von kulturellen und landschaftsschützenden gegenüber wirtschaftspolitischen Argumenten geführt, so haben sich die Dresdner eindeutig für eine aktive Wirtschaftsförderung entschieden: Durch den Verkehrszug sollen Wohn- und Gewerbegebiete miteinander verbunden und die innerstädtischen Verkehrswege entlastet werden. Außerdem erhoffen sich die Initiatoren des Bürgerentscheids, Dresdens Infrastruktur zu verbessern und seine Anziehungskraft als Wirtschaftsstandort zu steigern.35 Auch wenn das Projekt finanzpolitisch zunächst eine Ausgabe, freilich investiver Art darstellt, so kommt es doch den Interessen derjenigen, die der Demokratie Verschuldungsneigung und wirtschaftspolitischen Unverstand unterstellen, entgegen. Es sind vielmehr der Stadtrat und das Verwaltungsgericht, die in ihren Entscheidungsbegründungen auf die wirtschaftspolitischen Argumente gar nicht mehr eingehen. Dafür mögen gute Gründe sprechen: Widerlegt wird in jedem Falle aber die von wirtschaftsliberaler Seite gepflegte Unterstellung, Demokratie hemme optimierte Wirtschafts- und Finanzpolitik. ${ }^{6}$

Zwar anerkennt das Verwaltungsgericht in seinem Urteil die Unantastbarkeit eines Bürgerentscheids, sofern dieser nicht die verfassungsmäßige Ordnung dispensiert. Faktisch führt diese Achtung vor dem Souverän jedoch dazu, dass materielle Überlegungen ausschließlich dem Problem der UNESCO-Tauglichkeit einer Elbquerung gewidmet sind. Der erklärte Wille des Souveräns wird und kann nur in Gestalt von formellen, also verfahrensrechtlichen Argumenten erwogen werden. Denn Gegenstand verwaltungsgerichtlicher Kontrolle kann bekanntlich nur exekutives Handeln sein. Entscheidungsgegenstand sind somit das im (vom Rat angeordneten!) Widerspruch des Oberbürgermeisters liegende Suspensivinteresse und das in der Ersatzvornahme des Regierungspräsidiums konkretisierte Vollzugsinteresse, das vom Gericht als weniger gewichtig erachtet wird. 37

35 www.pro-waldschloesschenbruecke.de/fragen/fragen.htm (Abruf I 8. März 2007).

36 Damit soll nicht behauptet werden, dass die wirtschaftspolitisch motivierte Entscheidung gegenüber einer kultur- und umweltschützenden Entscheidung sich langfristig tatsächlich auch wirtschaftlich positiver ausgewirkt hätte. Das zu beurteilen ist nicht das Thema dieser Untersuchung. Vielmehr verdeutlicht diese Anmerkung zu den Motiven der einzelnen Akteure, in welchem Maße auch inhaltlich fragwürdig ist, was überhaupt die »sachlich bessere « Entscheidung darstellt.

37 Verwaltungsgericht Dresden AZ i 2 K I768/06, S. 8 ff. Zum Unterschied von Suspensiv- und Vollzugsinteresse: Alfred G. Debus, Überwiegendes Vollzugsinteresse wegen gesetzlichen Ausschlusses der aufschiebenden Wirkung?, Neue Zeitschrift für Verwaltungsrecht 2006, S. 49 ff. Auch nach dieser verwaltungsrechtlichen Perspektive, bei der, es sei noch einmal betont, das Regierungspräsidium Dresden, was in der Sache aber nur den Inhalt des Bürgerentscheids vertritt, Gegner ist, stellt sich das Suspensivinteresse keinesfalls zwingend als vorrangig dar. Vielmehr ist erst in den vergangenen zwanzig Jahren allmählich zwischen gesetzlichem und behördlichem Suspensionsausschluss unterschieden worden. Dem Suspensivinteresse wird teilweise sogar dann nachgegeben, wenn der Gesetzgeber ausdrücklich aufschiebende Wirkung eingelegter Rechtmittel ausschließt. Dies folgt dem hohen Wert, der in Deutschland dem Rechtsschutz des einzelnen Bürgers zugemessen wird, ebd., 49 und $5 \mathrm{I}$. Wird dies von der herrschenden Meinung »im zweipoligen Staat-Bürger-Verhältnis in Hinblick auf Art. I9 IV GG« als »geboten« erachtet, so ist im vorliegenden Beispiel erneut zu verinnerlichen, dass der Kläger die kommunale Exekutive und die dahinter stehende kommunale Legislative sind, also nicht ein schwacher Einzelbürger gegenüber einem hoffnungslos überlegenen Staat. Aber genau auf diese Konstellation hin ist das hohe Gut des Rechtsschutzes und der aufschiebenden Wirkung von Rechtsmitteln dimensioniert. Einer der häufigeren Fälle, bei denen dem Suspensivinteresse Vorrang zugebilligt wurde, waren somit auch inkriminierte Asylverfahren. 
Wie parlamentarische Aussetzung von Demokratie altes deutsches obrigkeitsstaatliches Denken revitalisiert und traditionelle Aversion gegenüber Parlamentarismus begünstigt, zeigt eine enttäuschte Klage eines derjenigen, die das Bürgerbehren dereinst initiiert haben: $3^{3}$

"Wir können froh sein, August den Starken gehabt zu haben, 39 sonst würde heute noch debattiert, was an die Stellen von Zwinger, Semperoper, Hofkirche etc. wohl gebaut werden sollte. [...] Damals verhinderten auch einige Stadträte die Vereinigung der zwei Städte rechts und links der Elbe. August ließ sie kurzerhand in Torgau einsperren, bis sie einsichtig wurden. $\ll^{40}$

Somit wird ein absolutistischer Monarch zum Anwalt des Volkes: Johann Sebastian Bachs auf den großen Wettiner komponierte Jubelkantaten spiegeln die Stimmung nicht weniger Dresdner auch nach über 200 Jahren anscheinend in ungebrochener Aktualität wider. Die Konstellation führt jene tragische deutsche Situation herbei, in der das Parlament als Gegner des Volkes und die Gerichte als dessen Anwalt wahrgenommen werden, was sie in diesem Falle ja noch nicht einmal waren. Neben dem Konflikt von direkter und parlamentarischer Demokratie weist das Beispiel jedoch noch einen weiteren Konflikt auf, nämlich einen innerhalb der repräsentativen demokratischen Institutionen: die Friktionen zwischen präsidialer Demokratie, durch den direkt gewählten Oberbürgermeister konkretisiert, und parlamentarischer Demokratie, in Gestalt des Stadtrats institutionalisiert. ${ }^{\mathrm{I}}$

Dieses Konfliktdreieck impliziert für den Historiker nahezu zwangsläufig die Parallele zur zweifelsohne kunstvollen Verfassung der Weimarer Republik. Lediglich die faktische Hierarchie ist im Falle Waldschlößchen umgekehrt: Desavouierte in Weimar der Reichspräsident das Parlament, so verhält es sich im vorliegenden Fall umgekehrt. Denn der Oberbürgermeister sieht sich genötigt, gegen seinen eigenen politischen Willen Widerspruch beim Regierungspräsidium einzulegen. Es ist indes eine Grundeinsicht der Psychologie, dass die Triade eine der kompliziertesten und konfliktgeneigtesten Konstellationen überhaupt ist. Bisweilen wird sie daher auch als »Dramadreieck« bezeichnet. Diese schlichte Weisheit findet in der Sächsischen Gemeindeordnung letztlich keine ausreichende Berücksichtigung. Vermutlich sind es die Erfahrungen der totalitären Vergangenheit, die hier zu einem überschießenden Ehrgeiz führten, allen gerecht $\mathrm{zu}$ werden..$^{2}$

38 Die zum klassischen deutschen obrigkeitsstaatlichen Denken anzutreffende Forschung ist kaum noch zu überschauen. Klassisch sind für das Paradigma der Weimarer Republik: Kurt Sontheimer, Antidemokratisches Denken in der Weimarer Republik. Die politischen Ideen des deutschen Nationalismus zwischen I91 8-1933, München 1992; Karl-Dietrich Bracher, Staatsbegriff und Demokratie in Deutschland, in: Politische Vierteljahresschrift 9 (I968), S. 2 ff.; Manfred Koch, Die Deutschen und ihr Staat. Ein Untersuchungsbericht, Hamburg 1972, S. 26 ff.; ferner: Ralf Zoll, Gemeinden als primärer Rahmen für politische Partizipation - Eine strukturelle Analyse, in: Ralf Zoll (Hrsg.), Vom Obrigkeitsstaat zur entgrenzten Politik. Politische Einstellungen und politisches Verhalten in der Bundesrepublik seit den sechziger Jahren, Wiesbaden I999, S. 30 ff. ( 38 ff.). Zu bedenken ist, dass in den Neuen Bundesländern dieser Wandlungsprozess frühestens mit der Wiedervereinigung hätte einsetzen können.

39 Friedrich August der Starke (* I 2. Mai I670, † I. Februar I733), ab I694 Kurfürst von Sachsen. Gilt als entscheidender Protagonist des absolutistischen Fürstenstaates in Sachsen.

40 Abruf http://www.pro-waldschloesschenbruecke.de/unterstuetzer/unterstuetzer.htm (30. Dezember 2006). S. auch Georg Mester, Die Volksinitiative in Sachsen. Ein Beitrag zur verfassungsgeschichtlichen und verfassungsrechtlichen Entwicklung des legislativen Einleitungsverfahrens seit der Verfassung von I 83 I, Frankfurt am Main 2003, v. a. S. I93.

4I Zu diesen beiden Demokratietypen: Lijphart (Fn. 28), S. 9 und ${ }_{104} \mathrm{ff}$.

42 Die konkreten Umstände der Entstehungsgeschichte des Rechtsinstitutes des Volksentscheides in Sachsen sind bei Mester (Fn. 40), S. I67 ff., dargestellt. 
Am Ende haben alle Beteiligten Schaden genommen: Stadtrat, Oberbürgermeister und die Bürger ohnehin. Relativ unbeschädigt bleiben die außerkommunalen, gleichsam sekundären Akteure dieses ursprünglich innerkommunalen Konflikts: die mittelbar über den Landtag demokratisch legitimierte Sächsische Staatsregierung und die de facto demokratisch gar nicht legitimierte Instanzenjudikative. Der Fall ist ein Lehrbuchbeispiel für die Selbstenthebelung von Demokratie. Nicht nur die Steuerungseffizienz, sondern auch die Steuerungseffektivität steigt offensichtlich in dem Maße, in dem die Anzahl unmittelbar demokratisch gewählter politischer Akteure und Aktionsformen reduziert ist. Was bleibt, ist ein Paradoxon: Seltener den Souverän zur Urne zu rufen heißt, ihm mehr Macht zu geben.

\section{Strafverfahren bei häuslicher Gewalt}

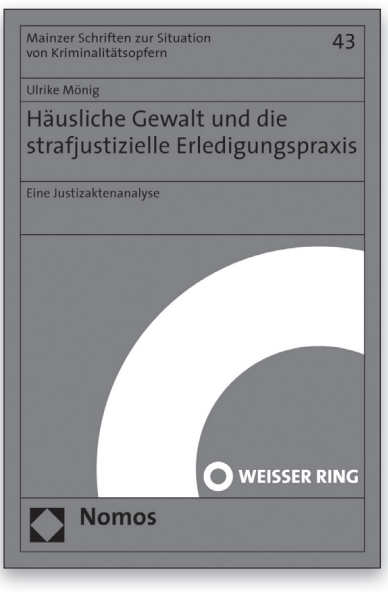

Häusliche Gewalt und die strafjustizielle Erledigungspraxis

Eine Justizaktenanalyse

Von Prof. Dr. Ulrike Mönig, FH Bielefeld

2007, 159 S., brosch., 28,- €, ISBN 978-3-8329-2504-8 (Mainzer Schriften zur Situation von Kriminalitätsopfern, Bd. 43)

Wie geht die Strafjustiz vor dem Hintergrund des neuen Gewaltschutzrechts und des damit angestrebten Paradigmenwechsels mit Verfahren wegen häuslicher Gewalt um? Die Untersuchung geht der Frage nach, auf welche Art und Weise bei der Polizei registrierte Vorgänge wegen häuslicher Gewalt im weiteren Verlauf des Strafverfahrens behandelt und abgeschlossen werden.
Bitte bestellen Sie bei Ihrer Buchhandlung oder bei Nomos Telefon 07221/2104-37 | Fax -43 | www.nomos.de | sabine.horn@nomos.de

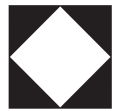

Nomos 